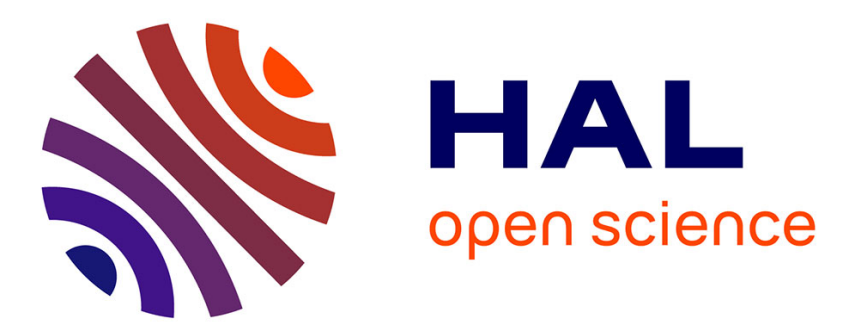

\title{
Robust control of a planar manipulator for flexible and contactless handling
}

Anne Delettre, Guillaume J. Laurent, Yassine Haddab, Nadine Le Fort-Piat

\section{To cite this version:}

Anne Delettre, Guillaume J. Laurent, Yassine Haddab, Nadine Le Fort-Piat. Robust control of a planar manipulator for flexible and contactless handling. Mechatronics, 2012, 22 (6), pp.852-861. 10.1016/j.mechatronics.2012.05.003 . hal-00734012

\section{HAL Id: hal-00734012 \\ https://hal.science/hal-00734012}

Submitted on 20 Sep 2012

HAL is a multi-disciplinary open access archive for the deposit and dissemination of scientific research documents, whether they are published or not. The documents may come from teaching and research institutions in France or abroad, or from public or private research centers.
L'archive ouverte pluridisciplinaire HAL, est destinée au dépôt et à la diffusion de documents scientifiques de niveau recherche, publiés ou non, émanant des établissements d'enseignement et de recherche français ou étrangers, des laboratoires publics ou privés. 


\title{
Robust control of a planar manipulator for flexible and contactless handling
}

\author{
Anne Delettre, Guillaume J. Laurent, Yassine Haddab, and Nadine Le Fort-Piat \\ Automatic Control and Micro-Mechatronic Systems Department, FEMTO-ST Institute, Université de Franche-Comté, ENSMM-UTBM-CNRS, \\ Besançon, France
}

\begin{abstract}
Many industries require non-contact and flexible manipulation systems, such as magnetic or pneumatic devices. In this paper, we describe a one-degree-of-freedom position control of an induced-air-flow surface. This device allows to convey objects on an air cushion using an original aerodynamic traction principle. A model of the system is established and the parameters are identified experimentally. A $H_{\infty}$ robust controller is designed and implemented on the device in order to control the object position. Experiments with objects of various dimensions and materials are conducted and showed the robustness capabilities of the controller.
\end{abstract}

Keywords: Air jet manipulation, non-contact transport, $H_{\infty}$ robust control

\section{Introduction}

Many industries require contactless transport and positioning of delicate or clean objects such as silicon wafers, glass sheets, solar cell or flat foodstuffs. The handling of delicate, freshly painted, hot, sensitive or micron-sized structured components is feasible because mechanical contact is avoided. Contamination from and of the end-effectors can be totally avoided. This can be important in food handling or in semiconductor production processes. Furthermore, dry friction forces are canceled, which enables accurate positioning or high velocity motions.

In order to avoid contact between feeding devices and work pieces, many handling approaches have been proposed. These methods typically employ magnetic [1] [2], electrostatic, near-field and aerodynamic levitation [3]. Both magnetic and electric levitation are restricted to conductive materials and the lifting force depends on material properties. Pneumatic levitation approaches use air flow to apply a force to a work piece. Because air flow is magnetic free, pneumatic approaches can be applied to any material: insulator or conductor, magnetic or non-magnetic, rigid or non-rigid.

Among the different techniques used to design contactless manipulators, aerodynamic levitation uses a flow of gas to apply a lift force which can be induced by Bernoulli levitation [4] [5] [6] or by an air cushion. Many devices using the last principle have been developed. They have been controlled in open loop and/or closed loop.

The MEMS array of Fukuta et al. [7] is composed of rows of microactuators. These actuators are used to create inclined air jets which allow conveying a plastic planar object in one direction with a maximum velocity of $8.3 \mathrm{~mm} . \mathrm{s}^{-1}$ in open loop control. Ku et al. [8] have used a converging wave technique to control the position of an object thanks to vertical tubes with blowing air or vacuum. Luntz and Moon [9] adapted the programmable vector fields of Bohringer [10] to their air-hockey table in addition to flow sinks (suction points) above the table. Thus they can move objects to a predictable orientation and position.

Different closed loop controllers have been designed to control the position of the object. In [8], a sequence of moving vacuum lines allows the object to reach the target position. Using an object detection, the authors can control three degrees of freedom. Fukuta et al. [7] have also made closed loop control with their device. They can make the object move from the left to the right of the surface by detecting the edge of the object and then activating the appropriate row of cells.

Konishi and Fujita [11] activate an intermittent air flow with a given pressure; the air flow is stopped when the object is near the target. The range of displacement of the micro silicon piece is $1 \mathrm{~mm}$ and can be completed in $200 \mathrm{~ms}$. 

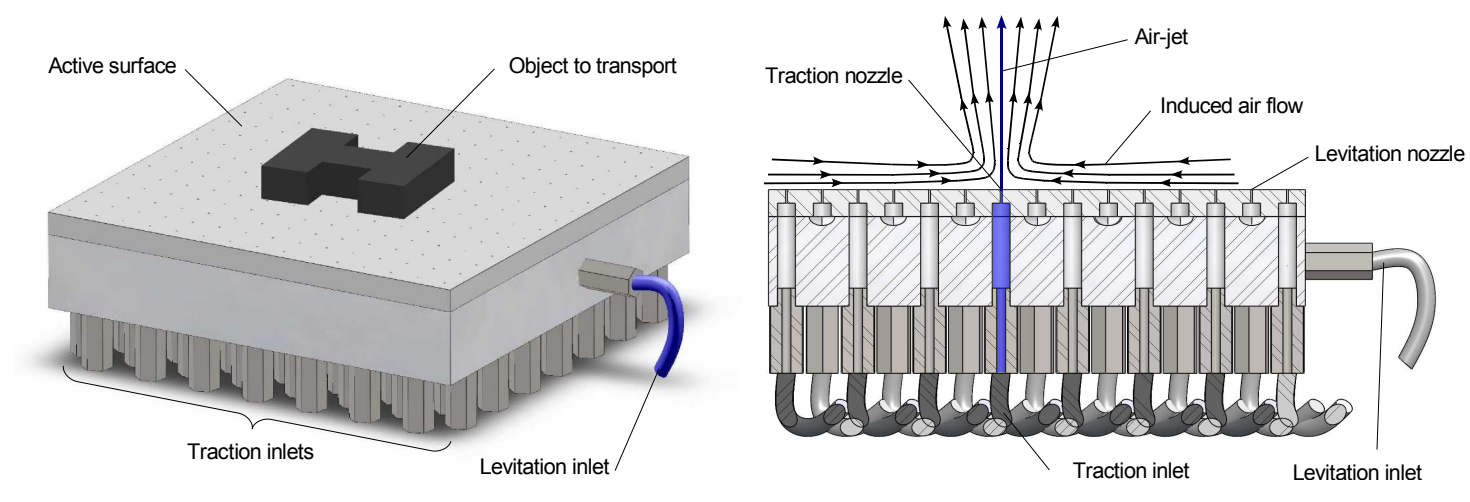

Figure 1: The induced air flow surface.

The Xerox PARK handling system [12] [13] [14] has demonstrated closed loop positioning accuracies in the order of $0.05 \mathrm{~mm}$ and trajectory tracking with typical velocity about $30 \mathrm{~mm} . \mathrm{s}^{-1}$. A proportional derivative controller is used and gives the forces and the torques to apply on the object. Then an actuator allocator determines the allocation for the air jets in order to produce the desired forces and torques.

In previous works [15], we proposed and described a new contactless conveyor. This device is an air hockey table which is able to convey objects using a traction principle and vertical air jets. We also experimented a PID controller in [16] which gave good results with one object (overshoot less than 5\% and settling time of $1.60 \mathrm{~s}$ ).

In all the works previously mentioned, only one kind of object was moved on each device. As many industries require conveying objects of different shapes, weights, or dimensions, the control must be robust to such object changes.

In this paper, the experiments consist in positioning six objects of different dimensions and materials with one degree of freedom ( $1 \mathrm{DOF})$. The $H_{\infty}$ method allows to implement a robust controller, to guaranty the stability of the closed loop and to deal with the non linearity of the system.

The paper is organized as follows. Part 2 presents a qualitative explanation of the operating principles and a description of the experimental device called induced air flow surface. Part 3 describes the choice of the control signal nature. In part 4, a model of the system is proposed. The parameter identification is reported in part 5. Finally, the control and the experimental results are presented in part 6.

\section{Induced air flow surface}

The induced air flow surface is a $120 \times 120 \mathrm{~mm}^{2}$ square surface on which an object can be lifted by aerodynamic levitation principle. The device consists of two parts detailed in the exploded view of Figure 1:

- the upper-block is drilled of $15 \times 15$ nozzles; each nozzle is $0.4 \mathrm{~mm}$ in diameter;

- the lower-block is drilled of 112 holes in staggered columns. These holes connect one hole out of two of the upper-block to independent air inlets; between the holes, a network of diagonal channels connects the other holes of the upper-block to a common air inlet located on the side of the lower-block.

The object is maintained in constant levitation thanks to an air cushion created by the airflow that comes through the lateral common air inlet. The airflow spreads over the network of diagonal channels and then through one hole out of two of the upper-block.

The object can be moved by generating strong vertical air-jets through the other holes of the surface. Each nozzle is driven by an independent solenoid valve ( $3 / 2$ normally closed valve). When a valve is open, air-flow comes through both blocks and generates a vertical air-jet on the front side of the upper-block. The air-jet creates an induced air flow in the surrounding fluid that pulls the object toward the nozzle (cf. Figure 1). This traction phenomena is a 


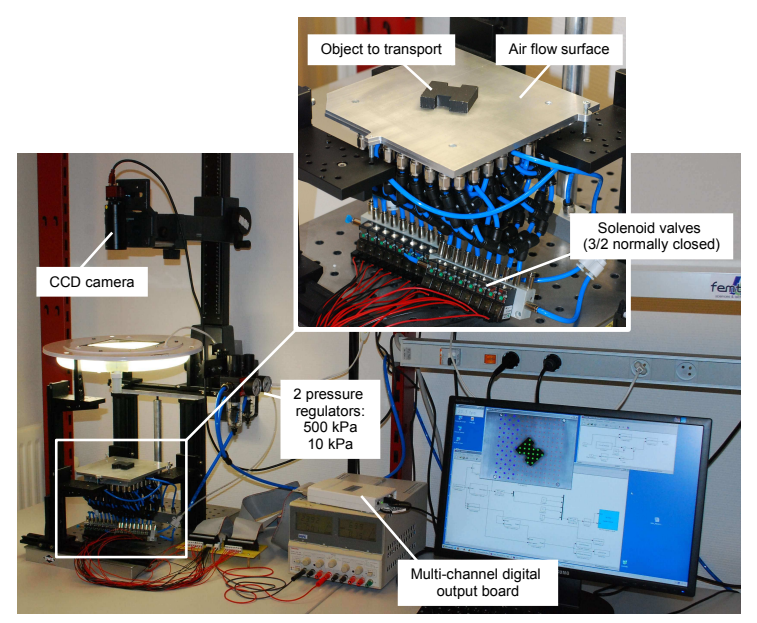

Figure 2: Overview of the experimental setup.

counter-intuitive physics demonstration of fluid mechanics such as the popular Bernoulli levitation [4].

The experimental setup for the distributed-air-jet manipulator is composed of pressurized air supply, two pressure regulators, the set of solenoid valves and its control system, and a computer for vision processing. The image taken by the camera is used to measure the position of the object that is used as feedback for the controller. Figure 2 shows the complete hardware configuration.

The induced air flow surface is put on a mechanical platform to adjust its equilibrium position. The air-flow source is provided by compressed air via two pressure control systems. One system controls the levitation and the other supplies air pressure for the valves. Default settings for operating pressures are $10 \mathrm{kPa}$ for levitation and $500 \mathrm{kPa}$ for traction.

Each valve is independently actuated by an electric signal. The electrical voltage applied to the valves is $24 \mathrm{~V}$ DC. Control signals are sent by computer via a multi-channel digital output board (NI USB-6509) from National Instruments and a $5 \mathrm{~V} / 24 \mathrm{~V}$ amplifier circuit.

A camera is used to grab video frames of the surface of the manipulator. The image processing is realized in the central process unit (CPU) of a computer at the rate of 60 frames per second (the software is OpenCV). Each image allows to measure the position of the object in real time.

\section{Control signal}

Our long term objective with this system is to control three degrees of freedom position of any object (translation in two-dimensions and in-plane rotation). In this paper, we focus on 1 DOF-control. For 1 DOF-moving, all the traction inlets of a same column are connected to the same valve. So every one valve is connected to every one column of holes of the lower block. Any combination of columns can be realized: a complete control signal is a vector of length 15 with values 0 (for a closed valve) or 1 (for an open valve). As some combinations produce almost the same effects on the object, we first selected those corresponding to the following properties:

- the active columns must be located in the same side of the object;

- the active columns must be consecutive;

- the columns located under the object and in the first column close to it cannot be opened. Indeed, an air jet flow induced by holes located next to the object would lift it and a complex behavior of the moving object would appear;

- the first active column close to the object cannot be farther than the fourth one. Indeed, if the first column is to far away, the effect on the object decreases rapidly and the air flow establishment time is too long. 

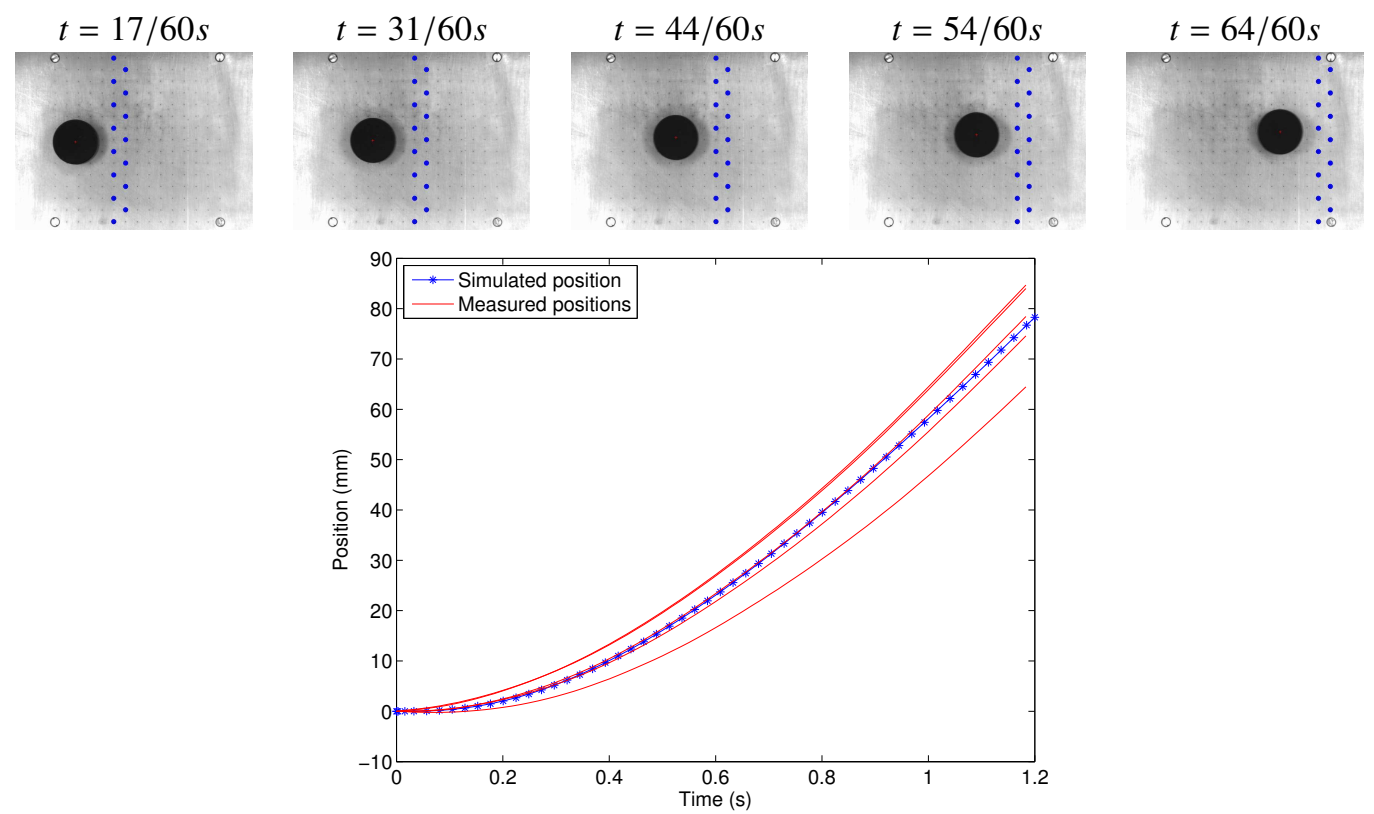

Figure 3: Image sequence and position versus time during open loop control experiments with two active columns and a distance value of 2. On the images, the big black circle is the object and the drawn dark small circles represent holes connected to open valves. On the plot, the experimental position is drawn for five different experiments and the model is estimated on the mean experimental position.

As shown in Figure 3, when two valves are open on the right, the object moves thanks to the air flow induced by the vertical air jets, in their direction. Thanks to the camera, the position of the object is measured and the two columns of active air jets next to the object are changing as the object is moving.

The object velocities at the exit of the active surface can be measured from position-vs-time curves. We have measured the object exit velocity for combinations of valves which verify the previous properties. These combinations are drawn on Figure 4. The number of active columns varies from one to five, and the distance between the object and the first active column is bigger than one and smaller than four times the distance between two nozzles. The corresponding measured exit velocities are shown in Figure 5. From these velocity measurements, we can note that the maximum range of velocities is achieved for a distance of 2 . A distance of 2 means that the first active hole column close to the object is the second one, i.e. the distance between the object and the closest air jet is between 8 and $16 \mathrm{~mm}$. Furthermore, the bigger the distance between the object and the active holes is, the longer the time of the induced air flow establishment is. As the control is then not reactive enough, we decide to define the control signal as the number of valves to open with distance 2. Figure 6 shows examples of the control signal which is positive if the active columns are located east of the object and negative if they are located west of the object. Note that the signal is an integer. Furthermore, it is saturated: the saturation depends on the physical limits of the surface and on the object position on it. We also choose to limit it to the range \pm 5 because air jets which are far away from the object have very small and slow effect on it.

\section{System identification}

In this section, a model of the whole system is established. To that aim, two sub-systems are distinguished: the valves and the surface on one hand, and the object on the other hand. A model of each one is proposed. All dimensions are in $\mathrm{mm}$.

The order of the system can be established thanks to the Strejc method [20,19] and from the variations of the position of the object as a function of time. Indeed, this graphical method uses the step response of a high order system. According to the Strejc method, a non-periodic system with $n$ different time constants can be approximated 


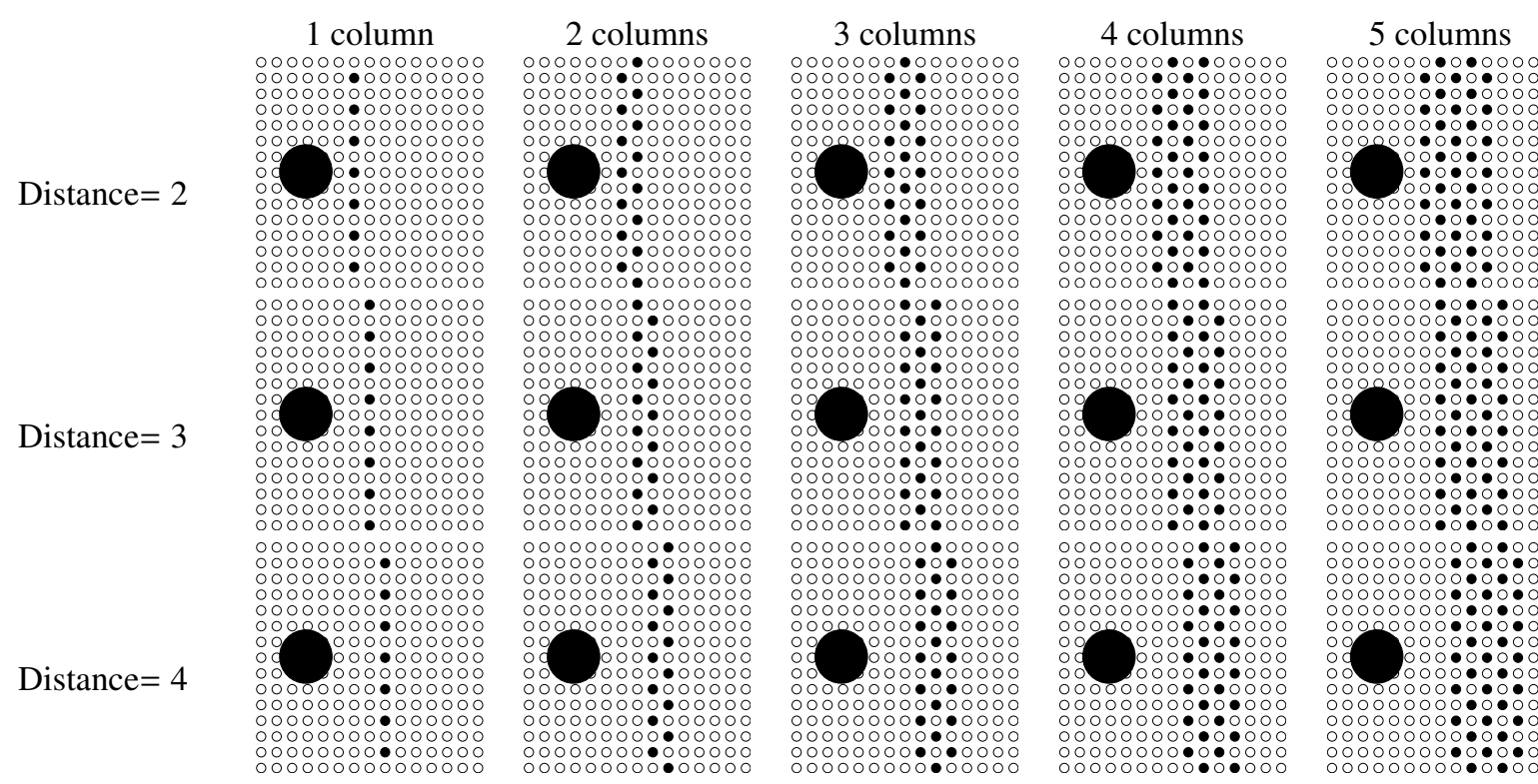

Figure 4: Different combinations depending on the number of active columns and their distance to the object. A small filled black circle represents a traction nozzle connected to an open valve and the big black circle the object to move.

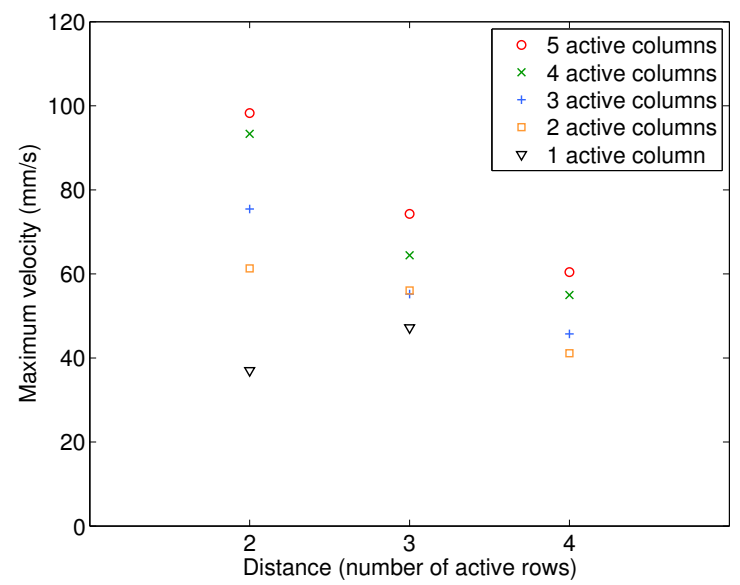

Figure 5: Object velocities at the exit of the surface, according to the number of active columns and their distance to it. A distance of 2 means that the first active column is the second one next to the object. All the active columns are consecutive.
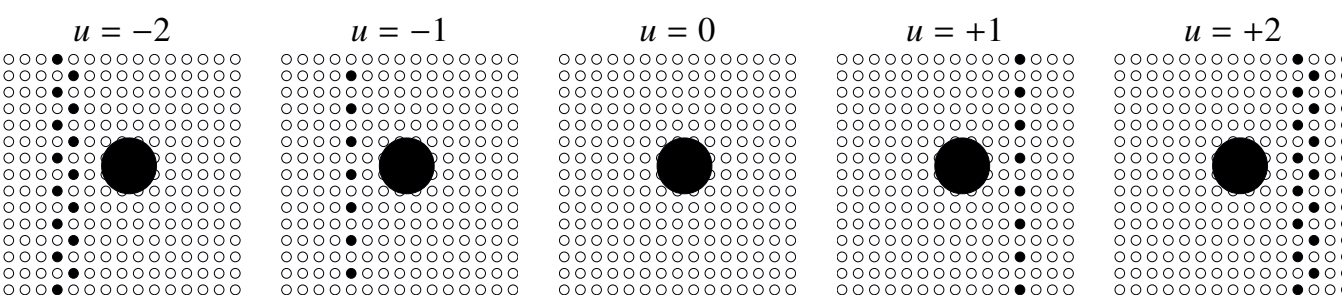

Figure 6: The control signal $u$ is an integer which represents the number of hole columns to open; it can be either positive or negative. A grey circle corresponds to a levitation nozzle, a small black one to a traction nozzle connected to an open valve and a white one to a closed valve. The big black circle represents the object to move. 
by a transfer function with $n$ identical time constants. From Figure 3, we can identify the system as a second order with an integrator.

Let's first establish the model of the object dynamic. To a first approximation, we can assume that the object receives two forces, a viscous traction due to the induced air flow and a viscous drag force due to friction of the air cushion on its backside:

$$
m \ddot{x}(t)=k_{t}\left[v_{\text {air }}(t)-\dot{x}(t)\right]-k_{d} \dot{x}(t)
$$

where $m$ is the mass of the object, $v_{\text {air }}$ is the velocity of the induced air flow, $k_{t}$ is the viscous traction coefficient corresponding to the front and lateral sides of the object (dimensions, shape, roughness, material), $k_{d}$ another viscous coefficient corresponding to the backside of the object (dimensions, shape, roughness, material) and $\dot{x}$ and $\ddot{x}$ respectively the velocity and the acceleration of the object.

In other words, the motion of the object is described by a first order model with integrator:

$$
\frac{X(s)}{V_{\text {air }}(s)}=\frac{\frac{k_{t}}{k_{t}+k_{d}}}{s\left(1+\frac{m}{k_{t}+k_{d}} s\right)}=\frac{K_{o}}{s\left(1+\tau_{o} s\right)}
$$

where $X(s)$ and $V_{\text {air }}(s)$ are the Laplace transform of respectively the position of the object and the air velocity.

As the system has been identified as a second order system with an integrator thanks to the Strejc method (detailed few lines before), and as the dynamic of the object is modeled by a first order with an integrator, we can model the induced air flow dynamics by a first order system:

$$
\frac{V_{a i r}(s)}{U(s)}=\frac{K_{a}}{1+\tau_{a} s}
$$

where $U(s)$ is the Laplace variable corresponding to the number of valves to open (as described in section 3 ), $K_{a}$ is the gain, and $\tau_{a}$ is the time constant corresponding to the establishment of the induced air flow including the dynamic of the valves.

The whole system can finally be modeled by:

$$
\frac{X(s)}{U(s)}=\frac{K_{o} * K_{a}}{s\left(1+\tau_{o} s\right)\left(1+\tau_{a} s\right)}
$$

\section{Parameters identification}

The parameters of the model have been identified experimentally using a mean square error minimization in the time domain.

First the parameter $\tau_{a}$ is identified from the measured air displacement. The air velocity measurement is done thanks to small expanded polystyrene balls. Indeed, by opening the valves, the balls move with the air flow and their velocity is approximately the same as the air flow because of their negligible mass. From the position of the balls for a step response (see Figure 7) we can identify $\tau_{a}$ : its value for a traction pressure of $500 \mathrm{kPa}$ is $\tau_{a}=0.085 \mathrm{~s}$.

Then, as the parameter $\tau_{o}$ depends on the object, it has to be identified for each object, from the curves of the object position as a function of time, under open loop control (example in Figure 5). The data for the six objects we use in this study are reported in Table 1 . Their geometrical and physical properties are also given in the Table.

And finally, the gains $K_{o}$ and $K_{a}$ have not been identified separately but together as a product. The identified values are also given in Table 1 for each object.

We can notice from these measurements that the gains for objects made of the same material are in the same order of magnitude.

\section{Control}

In order to control the X-position of the object on the surface, a $H_{\infty}$ robust controller is designed. 


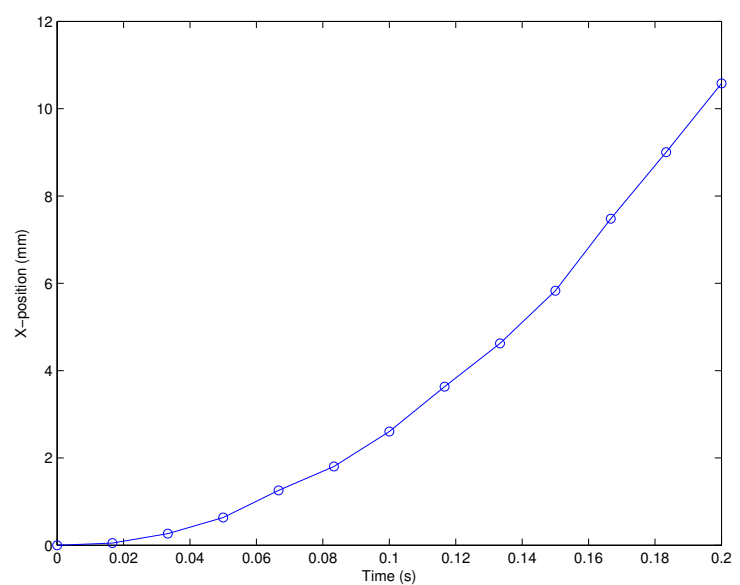

Figure 7: Displacement of a small polystyrene ball of negligible mass according to time. One valve is opened at time 0.

\begin{tabular}{|c|c|c|c|c|c|}
\hline Object Number & Material & Size $\emptyset \times \mathrm{xH}(\mathrm{mm})$ & Mass $(\mathrm{g})$ & $K_{a} K_{o}$ & $\tau_{0}(\mathrm{~s})$ \\
\hline 1 & Aluminum & $29.9 \times 14.80$ & 29.0 & 70.976 & 0.7982 \\
\hline 2 & Aluminum & $29.95 \times 5.03$ & 9.80 & 60.974 & 0.7666 \\
\hline 3 & Aluminum & $49.9 \times 12.10$ & 66.0 & 64.301 & 1.2745 \\
\hline 4 & Plastic & $30.01 \times 15.02$ & 9.57 & 113.19 & 0.4623 \\
\hline 5 & Plastic & $30.08 \times 5.15$ & 3.29 & 107.29 & 0.4660 \\
\hline 6 & Plastic & $48.5 \times 9.90$ & 16.4 & 113.61 & 0.9919 \\
\hline
\end{tabular}

Table 1: Characteristics of the objects. All of them are cylinders. 


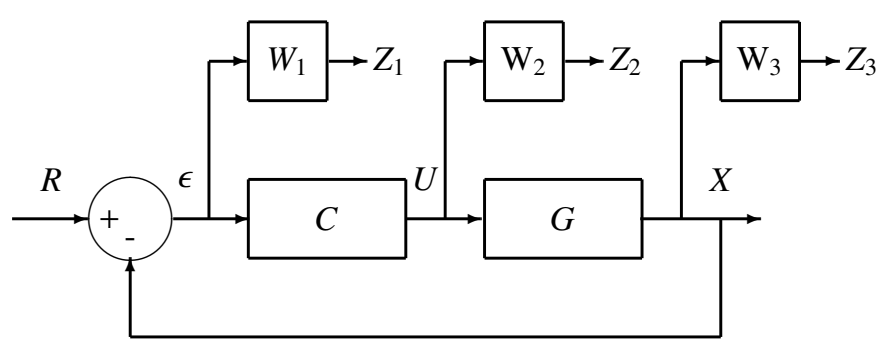

Figure 8: Diagram of the robust $H_{\infty}$ control. The controller and the system are represented respectively by $C$ and $G$. The weighting functions $W_{1}$, $W_{2}$ and $W_{3}$ are used to design the controller; the corresponding weighted outputs are $Z_{1}, Z_{2}$ and $Z_{3}$.

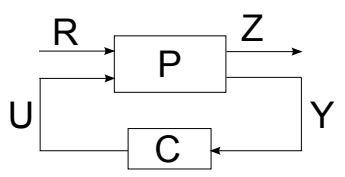

Figure 9: Standard form of the structure.

\section{1. $H_{\infty}$ robust method}

\subsubsection{Controller synthesis}

In order to have a good robustness of the controller against the change of objects, we use a method based on the $H_{\infty}$-norm [17]. Indeed, as we have seen in Table 1 (section 5), changes in physical and/or geometrical properties of the object lead to changes in the model parameters and then in the dynamics of the object. The $H_{\infty}$ controller design can take into account the parameter variations due to these changes. Furthermore, stability can be ensured for all the systems.

The structure we used to design the $H_{\infty}$ robust controller is shown in Figure 8.

The weighting functions $W_{1}, W_{2}$ and $W_{3}$ are designed to define the desired performances of the closed loop and the robustness of the controller. The error $\epsilon$ is weighted by the function $W_{1}$, the control signal $U$ by $W_{2}$ and the output of the system $X$ by $W_{3}$. The structure depicted in Fig. 8 can be equivalently represented by Fig. 9, where $P$ represents the system $G$ augmented with the weighting functions $W_{1}, W_{2}$ and $W_{3} . Y$ is the input of the controller $C$ and $U$ its output ; $R$ and $Z$ are respectively the inputs and the outputs of this system $P$.

The system $P$ can be represented by:

$$
\left[\begin{array}{l}
Z \\
Y
\end{array}\right]=\left[\begin{array}{ll}
P_{11} & P_{12} \\
P_{21} & P_{22}
\end{array}\right] \times\left[\begin{array}{l}
R \\
U
\end{array}\right]
$$

where $P_{11}, P_{12}, P_{21}$ and $P_{22}$ are the transfer from each input to each output of the system $P$. From Figure 9 , we can deduce the expression of $Z$ :

$$
Z=\left[\begin{array}{c}
Z_{1} \\
Z_{2} \\
Z_{3}
\end{array}\right]=\left[\begin{array}{cc}
W_{1} & -W_{1} G \\
0 & W_{2} \\
0 & W_{3} G
\end{array}\right] \times\left[\begin{array}{c}
R \\
U
\end{array}\right]
$$

where $G$ and $C$ are the transfer functions of the system and the controller respectively.

Assuming that: $U=C \epsilon$, Eq. 6 can be re-written:

$$
H=\left[\begin{array}{c}
W_{1} S \\
W_{2} C S \\
W_{3} T
\end{array}\right]
$$

where $H$ is the transfer function between the reference signal $R$ and the outputs $Z_{1}, Z_{2}$ and $Z_{3}, C$ is the transfer fonction of the controller, $S$ is the sensitivity function which corresponds to the transfer from the reference $R$ to the error $\epsilon$, and 
$T$ is the complementary sensitivity function. Their Laplace transforms $S(s)$ and $T(s)$ are given respectively by:

$$
\begin{gathered}
S(s)=\frac{\epsilon(s)}{R(s)}=\frac{1}{1+C(s) G(s)} \\
T(s)=\frac{X(s)}{R(s)}=1-S(s)
\end{gathered}
$$

where $\epsilon(s), R(s), C(s) G(s)$ and $X(s)$ are the Laplace transform of respectively $\epsilon, R, C G$ and $X$.

The aim of the $H_{\infty}$ robust method [18] is to define the controller $C$ that makes the closed loop stable in spite of bounded model variations (small-gain theorem) and that ensure the desired performances defined by the weighting functions. This is true if this relation is:

$$
\|H\|_{\infty} \leq \gamma
$$

with $\gamma=1$ and where the $H_{\infty}$-norm of a function is defined as its peak value:

$$
\|H\|_{\infty}=\sup _{\omega \in \mathbb{R}} \bar{\sigma}(H(j \omega))
$$

Here, the $H_{\infty}$-norm of the function $H$ is defined by:

$$
\|H\|_{\infty}=\left\|\begin{array}{c}
W_{1} S \\
W_{2} C S \\
W_{3} T
\end{array}\right\|_{\infty}
$$

The three weighting functions have been designed according to the wanted control performances and ensuring the stability of the closed loop.

From equations 12 and 10, the first function $W_{1}$ is used to define tracking performances by weighting the sensitivity function $S$ :

$$
\left|W_{1}(j \omega)\right| \leq \frac{\gamma}{|S(j \omega)|}
$$

We define the expected performances of the system by a second order system with transfer function:

$$
T_{d}(s)=\frac{X(s)}{R(s)}=\frac{0.999}{\frac{1}{\omega_{n}^{2}} s^{2}+\frac{2 \zeta}{\omega_{n}} s+1}
$$

where $T_{d}(s)$ is the Laplace transform of the desired complementary sensitivity function (closed loop transfer), $\omega_{n}$ is the natural frequency and $\zeta$ the damping ratio. The desired natural frequency $\omega_{n}=1.8 \mathrm{rad} . \mathrm{s}^{-1}$ and damping ratio $\zeta=0.8$ are tuned in order to have good performances: a small overshoot (smaller than $2 \%$ ) and an expected settling time less than $2 \mathrm{~s}$. The gain is set to 0.999 in order to avoid the infinite value of $\frac{1}{S_{d}}=\frac{1}{1-T_{d}}$.

The second weighting function $W_{2}$ limits the control signal $U$ sent to the system depending on the reference signal $R$ :

$$
\left|W_{2}(s)\right| \leq \frac{\gamma}{|C(j \omega) S(j \omega)|}
$$

We have set $W_{2}$ to the value 0.2 . It gives a sufficient signal to control the system, coupled with the quantization of the control signal (as explained before, the saturation of its absolute value to 5 is done thanks to an independent saturation block). This weighting function could have been designed as a more complex transfer function instead of a constant value in order to improve the performances of the controller, but the order of the controller would have been bigger 


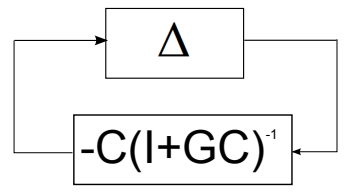

Figure 10: Equivalent diagram of the closed loop with multiplicative uncertainties.

too. As we will see next, this tuning of $W_{2}$ allows to design a controller which gives good performances.

Finally, the third weighting function $W_{3}$ ensures that the closed loop system remains stable in spite of variations in the model parameters. The change of the object properties causes such variations which can be bounded by the function $\Delta$ :

$$
\Delta=\frac{G-G_{0}}{G_{0}}
$$

where $G_{0}$ corresponds to the mean transfer function among the ones of the system modeled with each one of the six objects. It is the transfer function of the system when the object number 1 is used.

Assuming that the uncertainties $\Delta$ are multiplicative, the closed loop can be represented by the block diagram of Figure 10, where $G$ represents the transfer function of the system with object number 1 .

The condition for robust stability of the closed loop can be written, according to the small-gain theorem, as:

$$
\left\|\Delta C\left(I+G_{o} C\right)^{-1}\right\|_{\infty}<1 \Leftrightarrow\|\Delta T\|_{\infty}<1
$$

The robust stability condition (Eq. 17) can be re-written introducing the weighting function $W_{3}$. If $\Delta$ is bounded by $W_{3}$, we can write:

$$
\left\{\begin{array}{l}
\|\Delta\|_{\infty}<\left\|W_{3}\right\|_{\infty} \\
\left\|W_{3} T\right\|_{\infty}<\gamma
\end{array}\right.
$$

We have defined $W_{3}$ as follow, in order to verify Eq. 18 and to ensure the robust stability of the closed loop (see Bode magnitude plots in Fig. 11 (c) and (d)):

$$
W_{3}(s)=\left(\frac{s+1}{0.59 s+1}\right)^{2} * 0.65
$$

The $H_{\infty}$ method consists in finding a controller that ensures conditions of Eq. 13, 15 and 18 with a $\gamma$ value of 1 , that is to say that ensures the stability of the closed loop and the performances specified thanks to the filters $W_{1}, W_{2}$ and $W_{3}$. We have used the DGFK algorithm and Matlab to design this controller. No controller was found with a $\gamma$ value of 1, but a sub-optimal controller $C$ was found: it is a $7^{\text {th }}$ order system and the $\gamma$ value is 1.06 (this value will be discussed in next section). Its transfer function is given by:

$$
C(s)=\frac{2.029 E 2 s^{6}+4.388 E 3 s^{5}+2.99 E 4 s^{4}+8.317 E 4 s^{3}+1.013 E 5 s^{2}+4.499 E 4 s+0.9009}{s^{7}+1.724 E 2 s^{6}+8.474 E 3 s^{5}+2.228 E 5 s^{4}+1.443 E 6 s^{3}+3.331 E 6 s^{2}+2.394 E 6 s+2.690 E 3}
$$

Whereas the order of the system is quite high and could have been reduced neglecting the slow poles, we did not require to reduce it as the computer we used easily performed the calculus for the control in closed loop.

\subsubsection{Performances analysis}

The controller we have designed thanks to the $H_{\infty}$ method gives a $\gamma$ value higher than 1 . This means that at least one of the performances defined by the weighting functions $W_{1}, W_{2}$ and $W_{3}$ cannot be met. In order to check which 


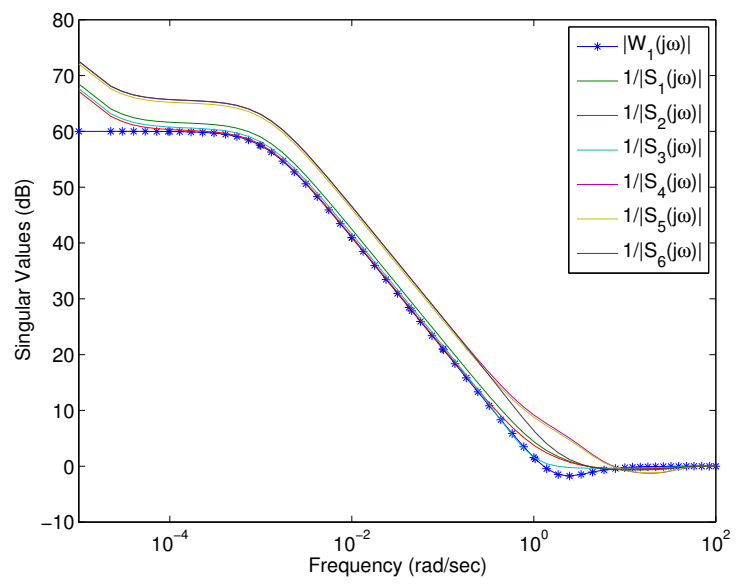

(a) Singular values of $W_{1}$ and $1 / S$ functions for each object.

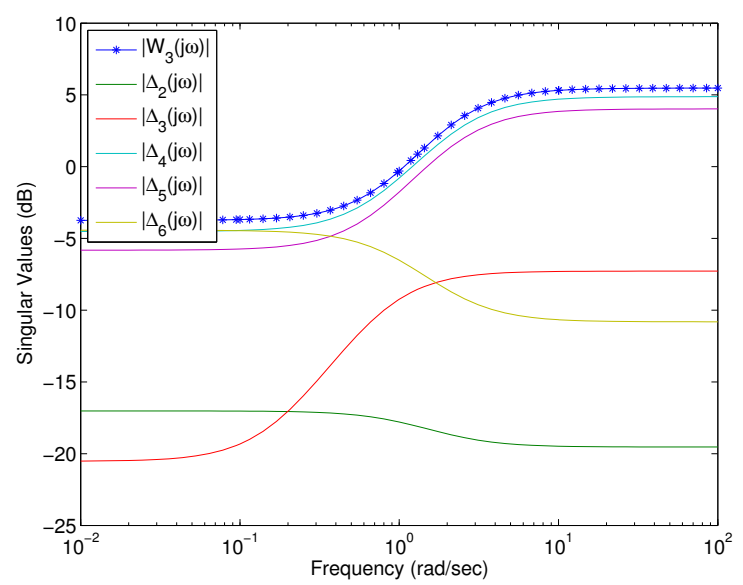

(c) Singular values of $W_{3}$ and $\Delta$ functions for each object (except the number 1 for which $\Delta=0$ ).

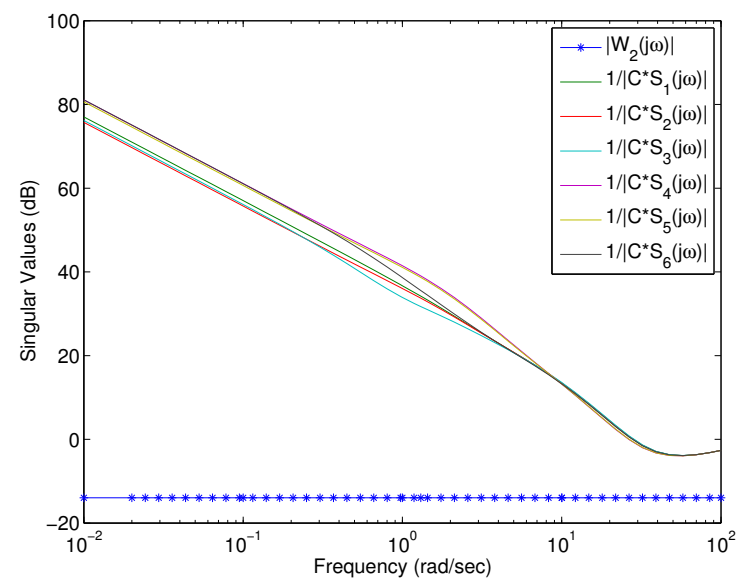

(b) Singular values of $W_{2}$ and $1 /(C S)$ functions for each object.

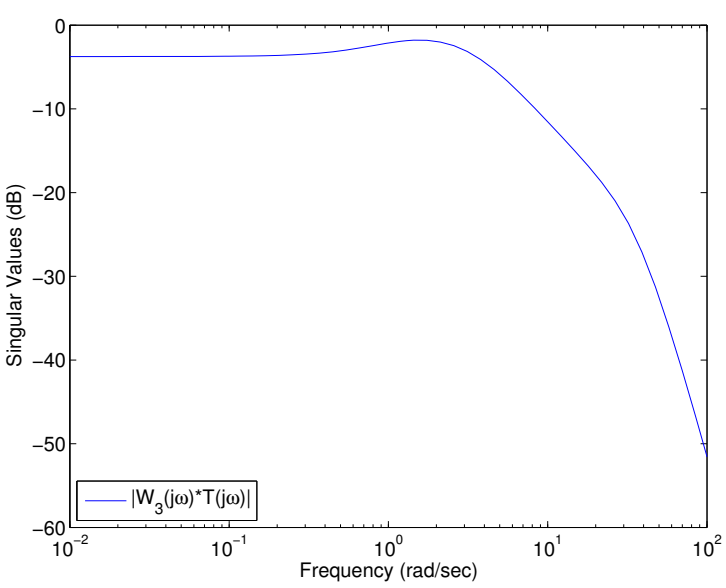

(d) Singular values of $W_{3} * T$ function.

Figure 11: Bode magnitude plots of the weighting functions $W_{1}, W_{2}$ and $W_{3}$, and of the appropriated functions to check the robustness conditions (Eq. 13, 15 and 18). 


\begin{tabular}{|c|c|c|c|c|}
\hline Object Number & Settling time 5\% $(\mathrm{s})$ & Rise time $(\mathrm{s})$ & Overshoot $(\%)$ & Static error $(\mathrm{mm})$ \\
\hline \hline 1 & 1.68 & 1.12 & 2.65 & $0.1110 / 0.0379 / 0.3631$ \\
\hline 2 & 1.83 & 1.23 & 2.51 & $0.0936 / 0.2419 / 0.1154$ \\
\hline 3 & 3.23 & 1.12 & 20.7 & $0.2202 / 0.4659 / 0.4044$ \\
\hline 4 & 1.87 & 1.33 & 0.70 & $0.6430 / 0.7105 / 0.3260$ \\
\hline 5 & 1.97 & 1.25 & 3.84 & $0.0854 / 0.9558 / 0.3931$ \\
\hline 6 & 1.70 & 1.20 & 0.78 & $0.5121 / 0.6662 / 0.2899$ \\
\hline
\end{tabular}

Table 2: Experimental performances of the $H_{\infty}$ robust controller with six different objects. All of them are measured on the first step, except the static error that is given for the three ones.

one(s) of the conditions defined in Eq. (13), (15) and (18) is/are true, we have plotted the bode magnitude plots of the appropriated functions in Fig. 11.

First, the (a) plot shows that the desired performances of the controller can not be exactly met (the $\gamma$ value is very close to 1). Indeed, the condition of Eq. 13 is not met for a gamma value of 1. Furthermore, the performances are better with the aluminum objects (number 1 to 3 ) than with the plastic ones (number 4 to 6 ) whose dynamics are faster.

Secondly, we can see on Fig. 11(b) that the condition on the control signal linked to Eq. 15 with a $\gamma$ value of 1 is true for all objects.

And finally, the condition of robust stability (Eq. 18) is also true for all objects. Indeed, we can see in Fig. 11(c) that the weighting function $W_{3}$ is higher than the $\Delta$ function for each object, and in Fig. 11(d), the singular values of the function $\left|W_{3} T\right|$ are lower than 1 for all the frequencies (so the peak value of the function $\left\|W_{3} T\right\|_{\infty}$ is also lower than 1). We can then conclude from the small-gain theorem (Eq. 17) that the closed loop is stable for all objects.

To conclude, the $\gamma$ value is higher than 1 because the performances defined by the weighting function $W_{1}$ cannot be met (settling time less than $2 \mathrm{~s}$ and overshoot smaller than 5\%). However the stability is guaranted for all objects.

\subsection{Experimental results}

Experimental control has been made with the six objects previously described and the $H_{\infty}$ robust controller designed in the last subsection.

During the experiments, the initial rest position of the object was maintained at 0 . Results are shown in Figure 12 and performances are summarized in Table 2. The performances are measured on the first reference step for each object ; the static error is also measured on the two last reference steps for each object.

The settling time is less than $2 \mathrm{~s}$ for all the objects except for the number 3 (because of an overshoot bigger than $5 \%$ ). During the experiments, whereas the overshoot was large for the third object (more than 20\%), it was less than $5 \%$ for the other ones. The mean value of the rise time is $1.21 \mathrm{~s}$ with a small standard deviation, and always less than $1.5 \mathrm{~s}$. The static error with the "mean object" used to design the controller (the object number 1) is around $0.1542 \mathrm{~mm}$ (it varies between 0.0379 and $0.9558 \mathrm{~mm}$ depending on the object and the reference step signal), and the repeatability (calculated as three times the standard deviation) is around $0.81 \mathrm{~mm}$ (the worst repeatability measured with the six objects is $2.29 \mathrm{~mm}$ ). Note that the choice of steps as reference signal allows to see the performances in the most unfavorable case. Taking a smooth reference signal would lead to better tracking.

The desired performances defined to design the controller are not totally met experimentally, as shown by a value of $\gamma=1.06$. Whereas the settling time is less than $2 \mathrm{~s}$, the overshoot is more than $2 \%$. We notice that the system is highly disturbed by ambient air flows. Rejection perturbation has not been taken into account during the controller design, but will be a part of future works. 


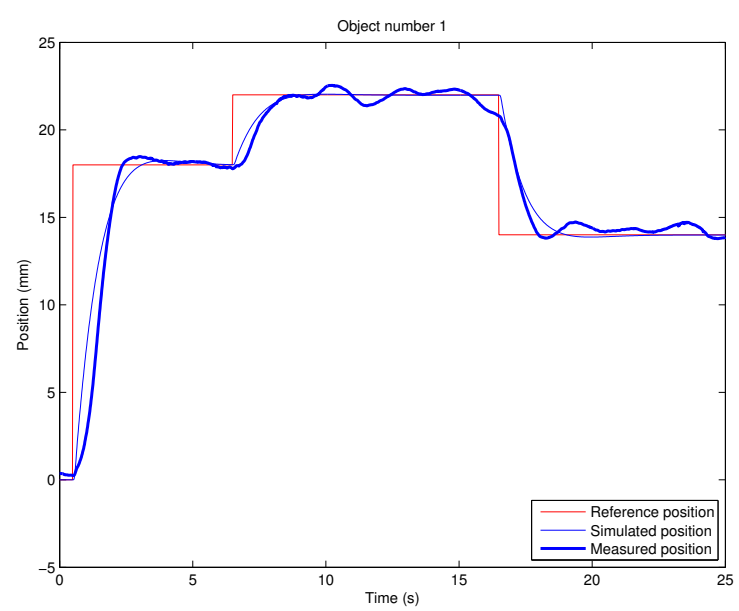

(a) Object Number 1

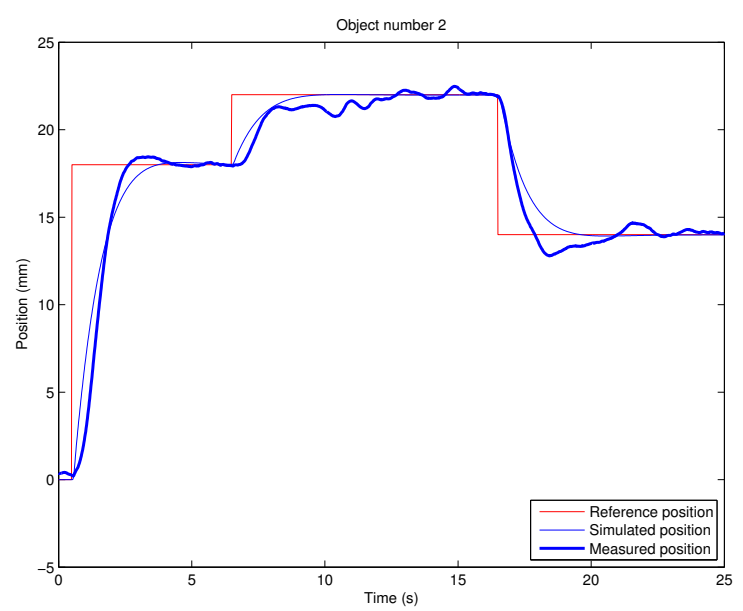

(c) Object Number 2

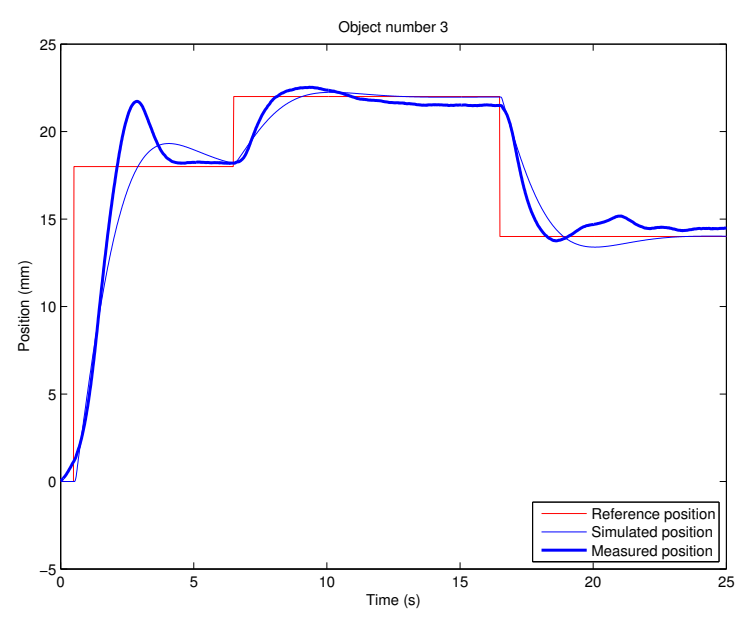

(e) Object Number 3

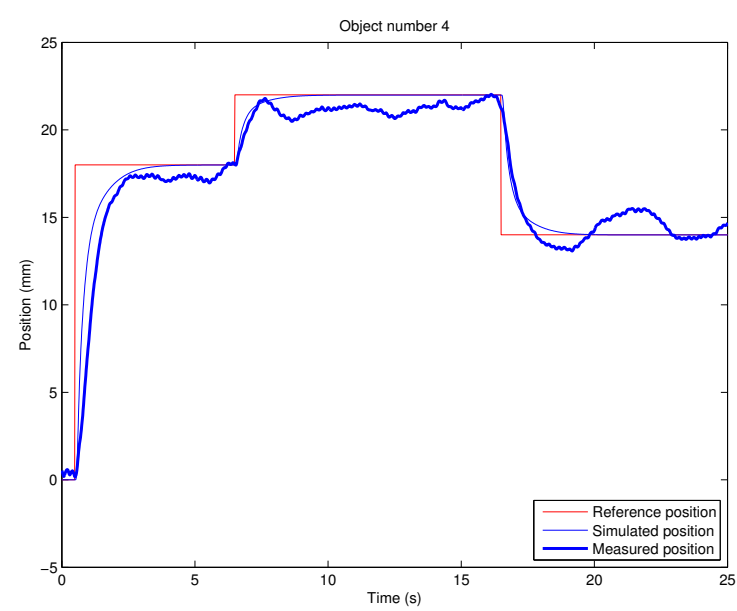

(b) Object Number 4

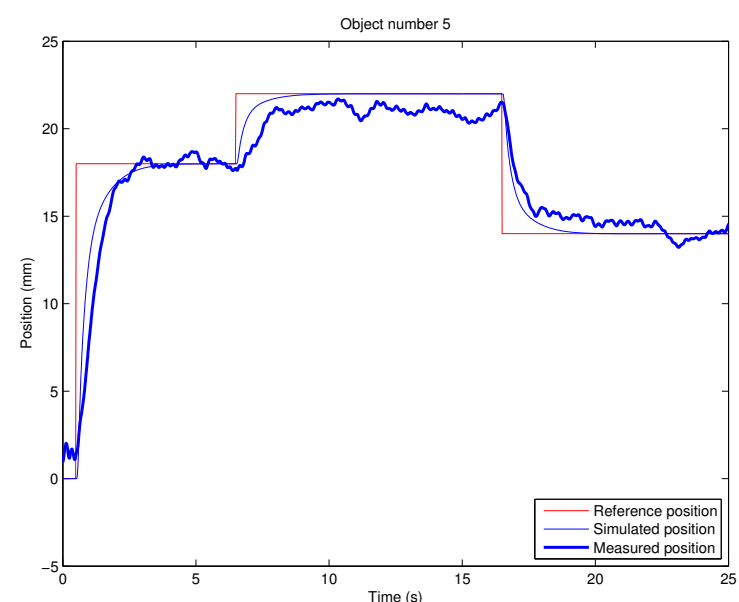

(d) Object Number 5

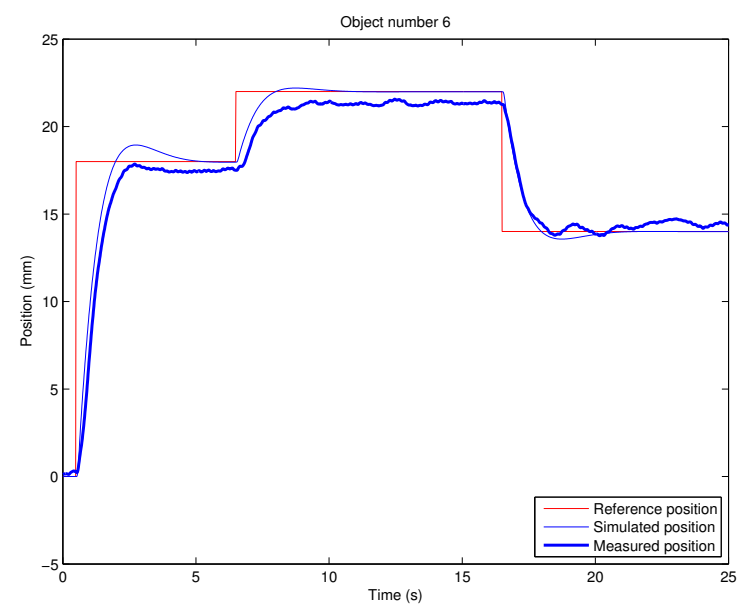

(f) Object Number 6

Figure 12: $H_{\infty}$ robust control results. For each object described in Table 1, the experimental position of the object and the simulated one are drawn as a function of time. The six objects descriptions are given in Table 1 . The simulation are done with the model of the system defined to design the $H_{\infty}$ controller. 


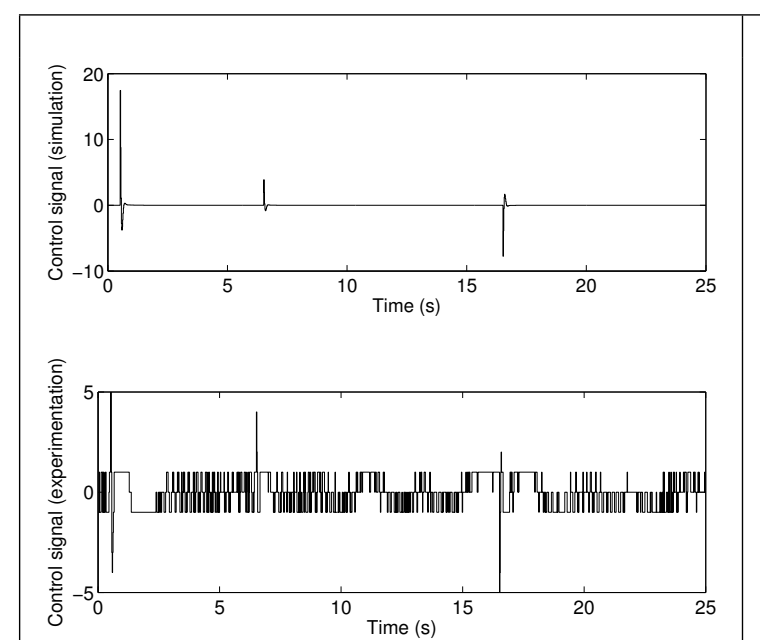

(a) Object number 1
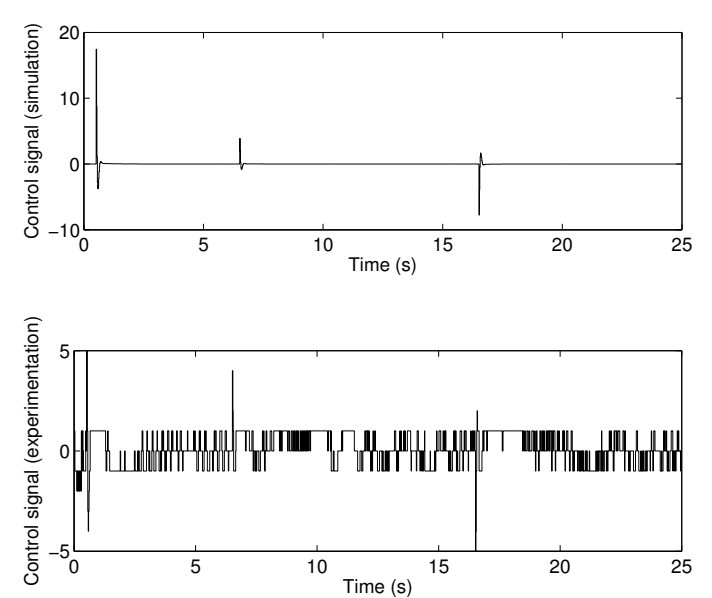

(c) Object number 3
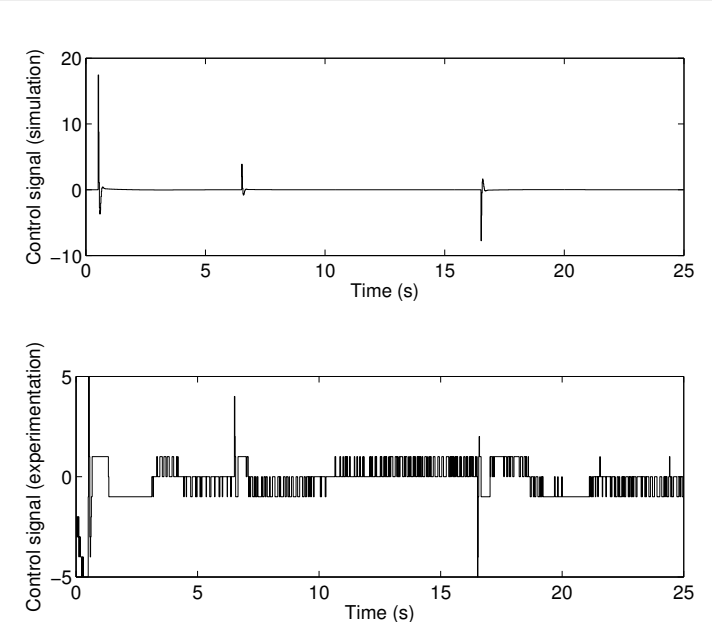

(e) Object number 5
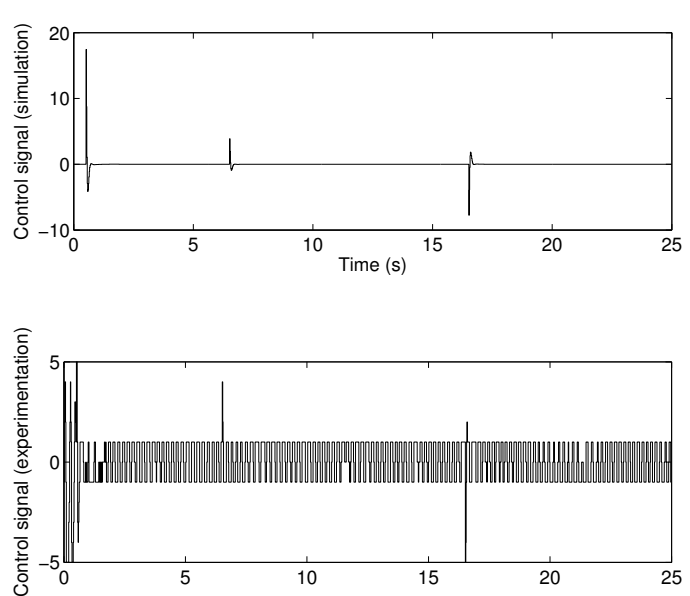

(b) Object number 2
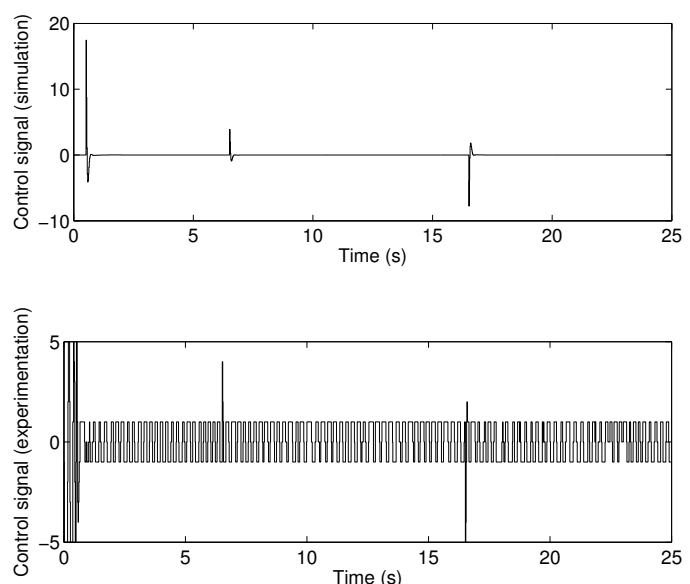

(d) Object number 4
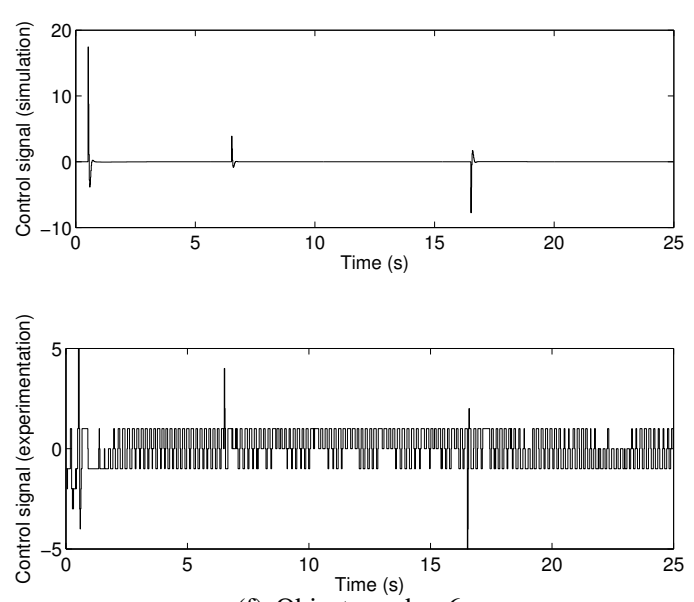

(f) Object number 6

Figure 13: Control signal sent to the system, with every one of the objects, and for both simulation and experimentation. 

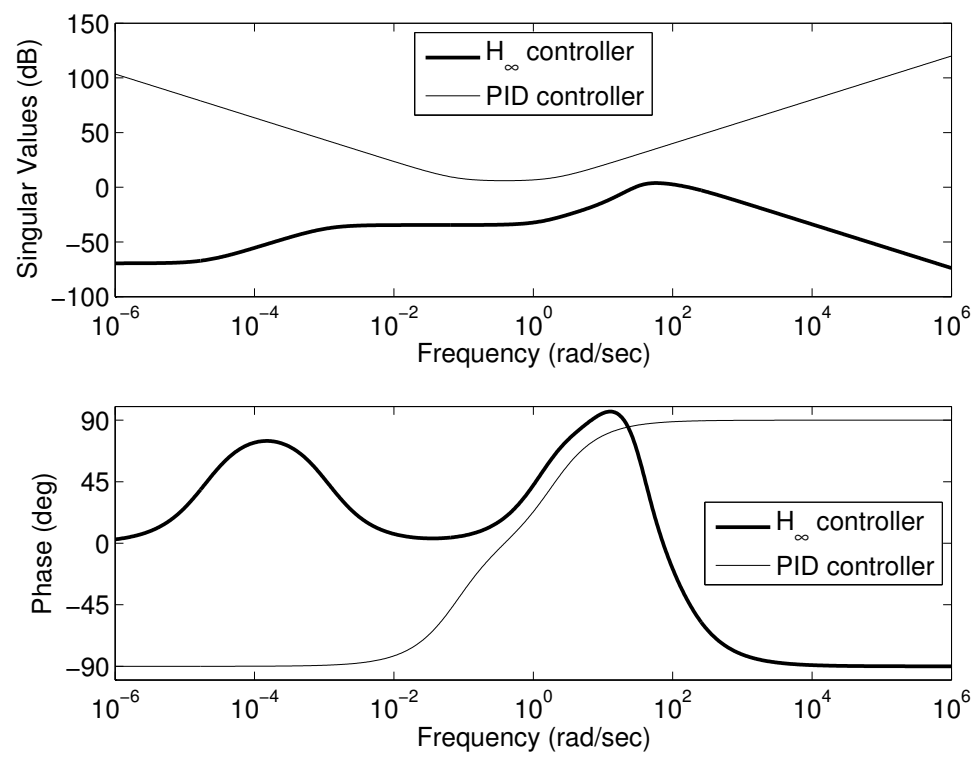

Figure 14: Bode diagram of both $H_{\infty}$ and PID [16] controllers.

\subsection{Discussion}

The experimental results are compared to the simulated ones in Fig. 12. The control signal sent to the system is also represented, in Fig. 13. Both experimental and simulated measurements curves are similar for the six objects. The model is not accurate but is sufficient to design a $H \infty$ controller that gives good performances.

We notice from the simulated results that overshoot can appear, especially with the largest objects (number 3 and 6). This overshoot also appears during experiments but is sometimes smaller during experiment than during simulation. We notice that it is coupled with a static error, what can be due to the quantization and the saturation of the control signal. Indeed, the $H_{\infty}$ method used to design the controller does not take into account the quantization nor the saturation of the control signal. We notice that the static error is bigger during the second step, what reinforces the influence of the quantization: the controller might take a long time to cancel the static error because of the quantized control signal.

Nevertheless the closed loop is stable with all the objects used, as predicted by theory.

If we compare these results with the one of previous works [16], the experimental results in closed loop control with the $H_{\infty}$ controller are better than with the PID controller designed before. The settling time was $1.6 s$ with the object number 1 , what is similar to the results with the $H_{\infty}$ controller, but the overshoot was more than $4 \%$ whereas it is $2.65 \%$ with the $H_{\infty}$ controller. As we can see on the bode diagram of both controllers (Fig. 14), the robust controller has lower gains and so the control signal is lower too. The solenoid valves are then less often activated than with the PID controller, but the response is then slower. Furthemore, as the robust controller is low-pass, it better filters the high frequencies than the PID controller. At lower frequency, the disturbance rejection from the $H_{\infty}$ robust controller is inferior to the PID controller, but the robustness is better.

Other objects of various shapes have also been tested and yielded good results ${ }^{1}$.

\section{Conclusion and future work}

In this paper, we have described and experimented a contactless conveyor based on an aerodynamic traction principle. A model of the system has been established for different kinds of objects and the parameters have been identified.

\footnotetext{
${ }^{1}$ This paper has supplementary downloadable material available at http://www.femto-st.fr/ guillaume.laurent/ provided by the authors.
} 
A robust controller has been tuned for $1 \mathrm{DOF}-$ position control and tracking. It gives good performances for all the objects tested experimentally, except for one object: overshoot less than 5\% and settling time less than $2 s$. As the method of controller design does not take into account the quantization and the saturation of the control signal, a specific controller that would consider them would give better performances.

Future work will consist in controlling three degrees of freedom of the object (two translations and one rotation). The 3D-manipulation will be possible if each traction air jet is individually controlled. Superimposing the effect of individually controlled air jets will allow to create appropriate forces and torques on the object to move it to the right position in a correct orientation. Both the model of the system and the control must then be distributed, what is a challenging problem.

\section{Acknowledgment}

This work was partially supported by the Smart Blocks NRA (French National Research Agency) project (ANR2011-BS03-005) and by the Nanorobust NRA project (ANR-2011-NANO-006). The authors gratefully acknowledge David Guibert and Joël Agnus from the FEMTO-ST Institute for their technical assistance.

\section{References}

[1] C. Elbuken, M. B. Khamesee, M. Yavuz, Design and Implementation of a Micromanipulation System Using a Magnetically Levitated MEMS Robot, IEEE/ASME Transactions on Mechatronics, 14 (2009) 434-445.

[2] K. S. Jung, Y. S. Baek, Study on a Novel Contact-Free Planar System Using Direct Drive DC Coils and Permanent Magnets, IEEE/ASME Transactions on Mechatronics, 7 (2002) 35-43.

[3] V. Vandaele, P. Lambert, A. Delchambre, Non-contact handling in microassembly: Acoustical levitation, Precision Engineering, 29 (2005) 491-505.

[4] C. Waltham, S. Bendall, A. Kotlicki, Bernoulli Levitation, American Journal of Physics, 71 (2003) 176-179.

[5] X. Li, K. Kawashima, T. Kagawa, Analysis of vortex levitation, Experimental Thermal and Fluid Science, 32 (2008) 1448-1454.

[6] S. Davis, J. Gray, D. G. Caldwell, An end effector based on the Bernoulli principle for handling sliced fruit and vegetables, Robotics and Computer-Integrated Manufacturing, 24 (2008) 249-257.

[7] Y. Fukuta, Y. A. Chapuis, Y. Mita, H. Fujita, Design, fabrication and control of MEMS-based actuator arrays for air-flow distributed micromanipulation, IEEE/ASME Journal of Microelectromechanical Systems, 15 (2006) 912-926.

[8] P. Ku, K. T. Winther, H. E. Stephanou, Distributed Control System for an Active Surface Device,IEEE International Conference on Robotics and Automation, 4 (2001) 3417-3422.

[9] J. E. Luntz, W. Messner, H. Choset, Distributed Manipulation Using Discrete Actuator Arrays, The International Journal of Robotics Research, 20 (2001) 553-583.

[10] K. Böhringer,B. R. Donald, R. Mihailovich, N. C. MacDonald, Sensorless manipulation using massively parallel microfabricated actuator arrays, IEEE International Conference on Robotics and Automation, 1 (1994) 826-833.

[11] S. Konishi, H. Fujita, A conveyance system using air flow based on the concept of distributed micro motion systems, IEEE/ASME Journal of Microelectromechanical Systems, 3 (1994) 54-58

[12] A. Berlin, D. Biegelsen, P. Cheung, M. Fromherz, D. Goldberg, W. Jackson, B. Preas, J. Reich, L. Swartz, Motion control of planar objects using large-area arrays of MEMS-like distributed manipulators, Presented at Micromechatronics'2000, (2000).

[13] D. K. Biegelsen, A. Berlin, P. Cheung, M. P. Fromherz, D. Goldberg, W. B. Jackson, B. Preas, J. Reich, L. Swartz, Air-jet Paper Mover, SPIE International Symposium on Micromachining and Microfabrication, (2000) 4176-11.

[14] W. B. Jackson, M. P. J. Fromherz, D. K. Biegelsen, J. Reich, D. Goldbergb, Constrained Optimization Based Control of Real Time Large-Scale Systems: Airjet Object Movement System, IEEE Conference on Decision and Control, 5 (2001) 4717-4720.

[15] G. J. Laurent, A. Delettre, N. Le Fort-Piat, A new aerodynamic traction principle for handling products on an air cushion, IEEE Transactions on robotics, 27 (2011) 379.

[16] A. Delettre, G. J. Laurent, N. Le Fort-Piat, A new contactless conveyor system for handling clean and delicate products using induced air flows, IEEE International Conference on Robots and Systems, (2010) 2351-2356.

[17] K. Zhou, K. Glover, J. Doyle, Robust and Optimal Control, Prentice Hall, 1996.

[18] H. Kwakernaak, Robust Control and $H_{i} n f t y$-Optimization - Tutorial Paper, Automatica, 29 (1993) 255-273.

[19] V. Strejc, Trends in identification, Automatica, 17 (1981) 7-21.

[20] V. Strejc, Approximation aperiodisscher ubertragungscharakteristiken, Regelungstechnik, 7 (1959) 124-128. 